PROCEEDINGS OF THE

AMERICAN MATHEMATICAL SOCIETY

Volume 125, Number 11, November 1997, Pages 3243-3253

S 0002-9939(97)03957-9

\title{
MEHLER-FOCK TRANSFORMS OF GENERALIZED FUNCTIONS VIA THE METHOD OF ADJOINTS
}

\author{
BENITO J. GONZÁLEZ AND EMILIO R. NEGRIN
}

(Communicated by Palle E. T. Jorgensen)

\begin{abstract}
In this paper we analyse the Mehler-Fock transform of generalized functions via the method of adjoints. For a distribution of compact support, we prove that its Mehler-Fock transform agrees with its transform via the kernel method. A Paley-Wiener type theorem is established.
\end{abstract}

\section{INTRODUCTION}

The Mehler-Fock transform of a distribution $f$ of compact support on $I$ (from now on $I=(0, \infty))$ was defined by the kernel method in [10] as:

$$
F(\tau)=\left\langle f(x), P_{-\frac{1}{2}+i \tau}(\cosh x)\right\rangle, \quad \tau>0,
$$

where, as it is usual, $P_{-\frac{1}{2}+i \tau}$ is the Legendre function of first kind and order zero.

Related studies on Mehler-Fock transforms of generalized functions have been carried out in [3], [4], [5], [8], amongst others.

In this paper, we study the Mehler-Fock transform by using the method of adjoints. For this purpose, it will be required to introduce two Fréchet spaces $V$ and $W$ satisfying the condition that the linear operator $\mathcal{L}: W \longrightarrow V$ given by

$$
(\mathcal{L} \psi)(x)=\int_{0}^{\infty} P_{-\frac{1}{2}+i \tau}(\cosh x) \psi(\tau) d \tau, \quad x>0
$$

be continuous.

Specifically, we denote by $V$ the vector space consisting of all complex-valued functions $\phi$ which possess derivatives of all orders on $I$ and such that, for all $k \in$ $\mathbb{N} \cup\{0\}$,

$$
\gamma_{k}(\phi)=\sup _{x \in I}\left|(\sinh x) A_{x}^{k} \phi(x)\right|<\infty,
$$

where $A_{x}$ is the differential operator

$$
A_{x} \equiv D_{x}^{2}+(\operatorname{coth} x) D_{x} \equiv(\sinh x)^{-1} D_{x}(\sinh x) D_{x}
$$

and $D_{x}$ denotes derivative with respect to the $x$-variable.

The topology of $V$ is that generated by the family of seminorms $\left\{\gamma_{k}\right\}_{k \in \mathbb{N} \cup\{0\}}$.

Received by the editors January 2, 1996 and, in revised form, April 24, 1996.

1991 Mathematics Subject Classification. Primary 44A15, 46F12.

Key words and phrases. Mehler-Fock transform, Legendre function, generalized functions, distributions of compact support, Paley-Wiener type theorem. 
Moreover, $W$ is the vector space of all complex-valued even functions $\psi$ on $\mathbb{R}$ such that

$$
\tau^{-1}\left(\frac{1}{4}+\tau^{2}\right)^{r} \cosh (\pi \tau) \psi(\tau) \in L^{1}(\mathbb{R})
$$

and

$$
\rho_{r}(\psi)=\sup _{x \in I}\left|x^{\frac{3}{2}} e^{x}\left(\mathcal{F}\left[\tau^{-1}\left(\frac{1}{4}+\tau^{2}\right)^{r} \cosh (\pi \tau) \psi(\tau)\right]\right)(x)\right|<\infty,
$$

for all $r \in \mathbb{N} \cup\{0\}$, and $\mathcal{F}$ denotes Fourier transform with respect to the $\tau$-variable. The space $W$ is equipped with the topology generated by the family of seminorms $\left\{\rho_{r}\right\}_{r \in \mathbb{N} \cup\{0\}}$.

As usual $V^{\prime}$ and $W^{\prime}$ denote the dual spaces of $V$ and $W$, respectively.

The Mehler-Fock transform of the generalized function $f \in V^{\prime}$ is given by $\mathcal{L}^{\prime} f$, where $\mathcal{L}^{\prime}$ is the adjoint of $\mathcal{L}$.

Here, we establish the link between these two methods. It will be proved that both definitions agree for distributions of compact support on $I$.

We also establish a Paley-Wiener type theorem. It is proved that every smooth function $F$ such that $|F(\tau)| \leq C \cdot\left(1+\tau^{2 r}\right)$, for all $\tau>0$, some nonnegative integer $r$ and some constant $C$, is the Mehler-Fock transform of a generalized function $f \in V^{\prime}$.

In this article we extend this analysis to the so-called Mehler-Fock transform of order $n \in \mathbb{N}$.

The corresponding results for the Kontorovich-Lebedev transform were carried out in [7].

\section{The OPERATOR $\mathcal{L}$ AND ITS ADJOINT}

The next result is the key to analysing the Mehler-Fock transform of generalized functions via the method of adjoints.

Theorem 2.1. The operator $\mathcal{L}$, given by $(1.2)$, is a continuous linear mapping from $W$ in $V$.

Proof. First observe that for all $\tau>0$,

$$
A_{x} P_{-\frac{1}{2}+i \tau}(\cosh x)=-\left(\frac{1}{4}+\tau^{2}\right) P_{-\frac{1}{2}+i \tau}(\cosh x) .
$$

So, for any $\psi \in W$ and all $k \in \mathbb{N} \cup\{0\}$, we have

$$
A_{x}^{k}(\mathcal{L} \psi)(x)=(-1)^{k} \int_{0}^{\infty}\left(\frac{1}{4}+\tau^{2}\right)^{k} P_{-\frac{1}{2}+i \tau}(\cosh x) \psi(\tau) d \tau .
$$

Using the integral representation (cf. [1, (11), p. 156]), which is valid for all $x>0$ and all $\tau>0$,

$$
P_{-\frac{1}{2}+i \tau}(\cosh x)=\frac{\sqrt{2}}{\pi} \cosh (\pi \tau) \int_{0}^{\infty}(\cosh x+\cosh \xi)^{-\frac{1}{2}} \cos (\tau \xi) d \xi
$$

and integrating (2.2) by parts, one obtains

$$
P_{-\frac{1}{2}+i \tau}(\cosh x)=\frac{\cosh (\pi \tau)}{\sqrt{2} \pi \tau} \int_{0}^{\infty}(\cosh x+\cosh \xi)^{-\frac{3}{2}}(\sin (\tau \xi))(\sinh \xi) d \xi .
$$


Substituting this expression in (2.1) and reversing the order of integration, it follows that

$$
\begin{aligned}
A_{x}^{k}(\mathcal{L} \psi)(x)=\frac{(-1)^{k}}{\sqrt{2} \pi} & \int_{0}^{\infty}(\cosh x+\cosh \xi)^{-\frac{3}{2}}(\sinh \xi) \\
& \times\left(\int_{0}^{\infty} \tau^{-1} \cosh (\pi \tau)\left(\frac{1}{4}+\tau^{2}\right)^{k} \psi(\tau) \sin (\tau \xi) d \tau\right) d \xi
\end{aligned}
$$

Note that the integral into the brackets is the Fourier sine transform of an odd function of the $\tau$-variable, and consequently it is the Fourier transform of this function.

Therefore,

$$
\begin{aligned}
A_{x}^{k}(\mathcal{L} \psi)(x)=\frac{(-1)^{k}}{\sqrt{2} \pi} \int_{0}^{\infty}( & \cosh x+\cosh \xi)^{-\frac{3}{2}}(\sinh \xi) \\
& \times\left(\mathcal{F}\left[\tau^{-1} \cosh (\pi \tau)\left(\frac{1}{4}+\tau^{2}\right)^{k} \psi(\tau)\right]\right)(\xi) d \xi
\end{aligned}
$$

Thus, for all $x>0$ we have

$$
\begin{aligned}
\left|(\sinh x) A_{x}^{k}(\mathcal{L} \psi)(x)\right|= & \mid \frac{\sinh x}{\sqrt{2} \pi(\cosh x)^{\frac{3}{2}}} \int_{0}^{\infty}\left(1+\frac{\cosh \xi}{\cosh x}\right)^{-\frac{3}{2}}(\sinh \xi) \\
& \times\left(\mathcal{F}\left[\tau^{-1} \cosh (\pi \tau)\left(\frac{1}{4}+\tau^{2}\right)^{k} \psi(\tau)\right]\right)(\xi) d \xi \mid
\end{aligned}
$$

which is less than or equal to

$$
\begin{aligned}
& \frac{1}{\sqrt{2} \pi} \int_{0}^{\infty} e^{-\xi} \xi^{-\frac{3}{2}}(\sinh \xi)\left|e^{\xi} \xi^{\frac{3}{2}}\left(\mathcal{F}\left[\tau^{-1} \cosh (\pi \tau)\left(\frac{1}{4}+\tau^{2}\right)^{k} \psi(\tau)\right]\right)(\xi)\right| d \xi \\
& \quad \leq M \rho_{k}(\psi),
\end{aligned}
$$

where $M$ is a suitable real constant. Thus, for all $k \in \mathbb{N} \cup\{0\}$,

$$
\gamma_{k}(\mathcal{L} \psi) \leq M \rho_{k}(\psi)
$$

This concludes the continuity of $\mathcal{L}$.

Now, the adjoint of the operator $\mathcal{L}$, which is given by

$$
\left\langle\mathcal{L}^{\prime} f, \psi\right\rangle=\langle f, \mathcal{L} \psi\rangle, \quad f \in V^{\prime}, \quad \psi \in W,
$$

is a continuous linear mapping from $V^{\prime}$ into $W^{\prime}$ when we provide $V^{\prime}$ and $W^{\prime}$ with their weak ${ }^{*}$-topologies. The Mehler-Fock transform of the generalized function $f$ is the member of $W^{\prime}$ given by $\mathcal{L}^{\prime} f$.

\section{THE LINK BETWEen Both Methods}

The next two lemmas allow us to establish the link between the two definitions of Mehler-Fock transforms of generalized functions.

For it, set

$$
\psi_{\phi}(\tau)=\tau \tanh (\pi \tau) \int_{0}^{\infty}(\sinh x) P_{-\frac{1}{2}+i \tau}(\cosh x) \phi(x) d x,
$$

for any $\phi \in V$ and all $\tau>0$. 
Lemma 3.1. For all $\phi \in V$ the function $\psi_{\phi}$ given by (3.1) satisfies

i) $\psi_{\phi}(\tau)=O\left(\tau^{2}\right)$, as $\tau \rightarrow 0$;

ii) for all $p \in \mathbb{N}, \psi_{\phi}(\tau)=O\left(\tau^{-p}\right)$, as $\tau \rightarrow \infty$.

Proof. i) Taking into account that $\tanh (\pi \tau)=O(\tau)$ for $\tau \rightarrow 0$, it follows that for $\tau$ small enough,

$$
\left|\psi_{\phi}(\tau)\right| \leq C_{1} \tau^{2} \int_{0}^{\infty}\left|(\sinh x) P_{-\frac{1}{2}+i \tau}(\cosh x) \phi(x)\right| d x \leq C_{2} \tau^{2},
$$

for some suitable real constants $C_{1}$ and $C_{2}$.

ii) If $A_{x}^{\prime}$ denotes the adjoint of $A_{x}$, namely, $A_{x}^{\prime}=D_{x}(\sinh x) D_{x}(\sinh x)^{-1}$, one has

$$
A_{x}^{\prime}\left[(\sinh x) P_{-\frac{1}{2}+i \tau}(\cosh x)\right]=-\left(\frac{1}{4}+\tau^{2}\right)(\sinh x) P_{-\frac{1}{2}+i \tau}(\cosh x),
$$

and so, for all $k \in \mathbb{N} \cup\{0\}$,

$$
(-1)^{k}\left(\frac{1}{4}+\tau^{2}\right)^{k} \psi_{\phi}(\tau)=\tau \tanh (\pi \tau) \int_{0}^{\infty}\left(A_{x}^{\prime}\right)^{k}\left[(\sinh x) P_{-\frac{1}{2}+i \tau}(\cosh x)\right] \phi(x) d x .
$$

Note that the integral in the right-hand side of (3.2) is equal to

$$
\int_{0}^{\infty}(\sinh x) P_{-\frac{1}{2}+i \tau}(\cosh x) A_{x}^{k} \phi(x) d x .
$$

In fact, integrating by parts,

$$
\begin{aligned}
\int_{0}^{\infty} A_{x}^{\prime} & {\left[(\sinh x) P_{-\frac{1}{2}+i \tau}(\cosh x)\right] \phi(x) d x } \\
= & \left.(\sinh x)\left(D_{x} P_{-\frac{1}{2}+i \tau}(\cosh x)\right) \phi(x)\right|_{0} ^{\infty} \\
& -\int_{0}^{\infty}(\sinh x)\left(D_{x} P_{-\frac{1}{2}+i \tau}(\cosh x)\right)\left(D_{x} \phi(x)\right) d x \\
= & \left.(\sinh x)\left(D_{x} P_{-\frac{1}{2}+i \tau}(\cosh x)\right) \phi(x)\right|_{0} ^{\infty} \\
& -\left.(\sinh x) P_{-\frac{1}{2}+i \tau}(\cosh x)\left(D_{x} \phi(x)\right)\right|_{0} ^{\infty} \\
& +\int_{0}^{\infty}(\sinh x) P_{-\frac{1}{2}+i \tau}(\cosh x) A_{x} \phi(x) d x .
\end{aligned}
$$

Observe that the remainder terms of the above integration by parts tend to zero as $x \rightarrow 0$ and $x \rightarrow \infty$. Indeed, since $\phi \in V$, it follows that $(\sinh x) \phi(x)$ is bounded on $I$, and

$$
D_{x} P_{-\frac{1}{2}+i \tau}(\cosh x) \longrightarrow 0, \text { as } x \rightarrow 0 \text { and } x \rightarrow \infty .
$$

Hence,

$$
(\sinh x)\left(D_{x} P_{-\frac{1}{2}+i \tau}(\cosh x)\right) \phi(x) \longrightarrow 0, \text { as } x \rightarrow 0 \text { and } x \rightarrow \infty .
$$

Also, since for all $\phi \in V$, the function $(\sinh x) D_{x} \phi(x)$ is bounded on $I$, and taking into account that $P_{-\frac{1}{2}+i \tau}(\cosh x)=O\left(e^{-\frac{x}{2}}\right)$ as $x \rightarrow \infty$, it follows that

$$
(\sinh x) P_{-\frac{1}{2}+i \tau}(\cosh x) D_{x} \phi(x) \longrightarrow 0, \text { as } x \rightarrow \infty .
$$


On the other hand, for any $\phi \in V$ one has

$$
(\sinh x) D_{x} \phi(x)=O(x), \text { as } x \rightarrow 0 .
$$

Thus,

$$
(\sinh x) P_{-\frac{1}{2}+i \tau}(\cosh x) D_{x} \phi(x) \longrightarrow 0, \text { as } x \rightarrow 0 .
$$

This proves that

$$
-\left(\frac{1}{4}+\tau^{2}\right) \psi_{\phi}(\tau)=\tau \tanh (\pi \tau) \int_{0}^{\infty}(\sinh x) P_{-\frac{1}{2}+i \tau}(\cosh x) A_{x} \phi(x) d x .
$$

Now, for all $k \in \mathbb{N}, k>1$, one obtains

$$
\begin{gathered}
\int_{0}^{\infty}\left(A_{x}^{\prime}\right)^{k}\left[(\sinh x) P_{-\frac{1}{2}+i \tau}(\cosh x)\right] \phi(x) d x \\
=(-1)^{k-1}\left(\frac{1}{4}+\tau^{2}\right)^{k-1} \int_{0}^{\infty} A_{x}^{\prime}\left[(\sinh x) P_{-\frac{1}{2}+i \tau}(\cosh x)\right] \phi(x) d x \\
=(-1)^{k-1}\left(\frac{1}{4}+\tau^{2}\right)^{k-1} \int_{0}^{\infty}(\sinh x) P_{-\frac{1}{2}+i \tau}(\cosh x) A_{x} \phi(x) d x
\end{gathered}
$$

Iterating this process one arrives at

$$
(-1)^{k}\left(\frac{1}{4}+\tau^{2}\right)^{k} \psi_{\phi}(\tau)=\tau \tanh (\pi \tau) \int_{0}^{\infty}(\sinh x) P_{-\frac{1}{2}+i \tau}(\cosh x) A_{x}^{k} \phi(x) d x .
$$

Taking into account that $\phi \in V$ and the asymptotic behavior of $P_{-\frac{1}{2}+i \tau}(\cosh x)$ as $x \rightarrow 0$ and $x \rightarrow \infty$, one concludes that

$$
\left(\frac{1}{4}+\tau^{2}\right)^{k}\left|\psi_{\phi}(\tau)\right| \leq M \tau, \quad \forall \tau>0,
$$

for some suitable nonnegative constant $M$. Thus, the lemma holds.

Lemma 3.2. For all $\psi \in W$, there exists $\phi \in V$ such that $\psi=\psi_{\phi}$, where $\psi_{\phi}$ is given by $(3.1)$.

Proof. Let $\psi \in W$ and set $\phi=\mathcal{L} \psi$. Note that, for $x>0$, one has

$$
\begin{gathered}
\left(\mathcal{L} \psi_{\phi}\right)(x)=\int_{0}^{\infty} P_{-\frac{1}{2}+i \tau}(\cosh x) \psi_{\phi}(\tau) d \tau \\
=\int_{0}^{\infty} P_{-\frac{1}{2}+i \tau}(\cosh x) \tau \tanh (\pi \tau) \int_{0}^{\infty}(\sinh t) P_{-\frac{1}{2}+i \tau}(\cosh t) \phi(t) d t d \tau,
\end{gathered}
$$

and from the inversion formula of the Mehler-Fock transform, the above expression is equal to $\phi(x)$, which holds whenever $\phi(x) e^{\frac{x}{2}} \in L^{1}(0, \infty)$ (cf. [2]). Since $\phi \in V$, this condition is satisfied.

Thus,

$$
\mathcal{L} \psi_{\phi}=\phi=\mathcal{L} \psi
$$

Now, we will establish that $\mathcal{L}$ is one to one. For it, assume that $\mathcal{L} \psi=0$. Taking $k=0$ in (2.4) one has for all $x>0$,

$(\mathcal{L} \psi)(x)=\frac{1}{\sqrt{2} \pi} \int_{0}^{\infty}(\cosh x+\cosh \xi)^{-\frac{3}{2}}(\sinh \xi)\left(\mathcal{F}\left[\tau^{-1} \cosh (\pi \tau) \psi(\tau)\right]\right)(\xi) d \xi=0$. 
The change of variables $t+1=\cosh \xi$ yields

$$
0=(\mathcal{L} \psi)(x)=\frac{1}{\sqrt{2} \pi} \int_{0}^{\infty}(\cosh x+1+t)^{-\frac{3}{2}} \Psi(t) d t, \quad \forall x>0,
$$

where

$$
\Psi(t)=\left(\mathcal{F}\left[\tau^{-1} \cosh (\pi \tau) \psi(\tau)\right]\right)\left(\log \left(t+1+\sqrt{t^{2}+2 t}\right)\right) .
$$

Finally, since

$$
\int_{0}^{\infty}(\cosh x+1+t)^{-\frac{3}{2}} \Psi(t) d t
$$

is the generalized Stieltjes transform of the function $\Psi$, from its inversion formula (cf. $[9$, p. 180]) it follows that $\Psi \equiv 0$. Therefore,

$$
\left(\mathcal{F}\left[\tau^{-1} \cosh (\pi \tau) \psi(\tau)\right]\right)\left(\log \left(t+1+\sqrt{t^{2}+2 t}\right)\right)=0, \quad \forall t>0,
$$

whence $\psi \equiv 0$.

Theorem 3.1. Let $f \in \mathcal{E}^{\prime}(I)$. Then, for any $\psi \in W$, one has

$$
\left\langle\mathcal{L}^{\prime} f, \psi\right\rangle=\int_{0}^{\infty} F(\tau) \psi(\tau) d \tau
$$

where the function $F(\tau)$ is given by (1.1).

Proof. First, observe that $\mathcal{D}(I) \subset V \subset \mathcal{E}(I)$ and the topology of $V$ is stronger than the one induced on it by $\mathcal{E}(I)$. Thus, we may regard $\mathcal{E}^{\prime}(I)$ as a vector subspace of $V^{\prime}$.

By [6, Proposition 2, p. 97], the function $F(\tau)$ satisfies

i) For $\tau \rightarrow 0$,

$$
F(\tau)=O(1)
$$

ii) there exists $r \in \mathbb{N} \cup\{0\}$ such that for $\tau \rightarrow \infty$,

$$
F(\tau)=O\left(\tau^{2 r}\right) .
$$

Using Lemma 3.1 and Lemma 3.2 it follows that for any $\psi \in W$ one has

i) $\psi(\tau)=O\left(\tau^{2}\right)$, as $\tau \rightarrow 0$;

ii) for all $p \in \mathbb{N}, \psi(\tau)=O\left(\tau^{-p}\right)$, as $\tau \rightarrow \infty$.

Thus, the integral of the right-hand side of (3.3) makes sense.

Clearly, if $f \in \mathcal{D}(I)$, the result of this theorem holds. In fact, assume that $f$ has its support contained in the closed interval $[a, b] \subset I$; then, using Fubini's theorem, one has

$$
\begin{gathered}
\left\langle\mathcal{L}^{\prime} f, \psi\right\rangle=\langle f, \mathcal{L} \psi\rangle \\
=\int_{a}^{b} f(x)(\mathcal{L} \psi)(x) d x=\int_{a}^{b} f(x) \int_{0}^{\infty} P_{-\frac{1}{2}+i \tau}(\cosh x) \psi(\tau) d \tau d x \\
=\int_{0}^{\infty} \psi(\tau) \int_{a}^{b} f(x) P_{-\frac{1}{2}+i \tau}(\cosh x) d x d \tau=\int_{0}^{\infty} F(\tau) \psi(\tau) d \tau .
\end{gathered}
$$

So, the result holds for $f \in \mathcal{D}(I)$.

Now, for $f \in \mathcal{E}^{\prime}(I)$ and using [11, Theorem 28.2(i), p. 301], there exists a sequence of functions $\left\{f_{m}\right\}_{m \in \mathbb{N}}$ belonging to $\mathcal{D}(I)$ which converges to $f$ in $\mathcal{E}^{\prime}(I)$, 
and hence, in $V^{\prime}$. Furthermore, from Theorem 2.1 it follows that $\mathcal{L}^{\prime}$ is a continuous mapping from $V^{\prime}$ into $W^{\prime}$. Thus $\left\{\mathcal{L}^{\prime} f_{m}\right\}_{m \in \mathbb{N}}$ converges to $\mathcal{L}^{\prime} f$ in $W^{\prime}$ as $m \rightarrow \infty$.

Since, for all $\psi \in W$,

$$
\left\langle\mathcal{L}^{\prime} f_{m}, \psi\right\rangle=\int_{0}^{\infty} F_{m}(\tau) \psi(\tau) d \tau
$$

where

$$
F_{m}(\tau)=\left\langle f_{m}(x), P_{-\frac{1}{2}+i \tau}(\cosh x)\right\rangle
$$

it follows that

$$
\left\langle\mathcal{L}^{\prime} f, \psi\right\rangle=\lim _{m \rightarrow \infty} \int_{0}^{\infty} F_{m}(\tau) \psi(\tau) d \tau .
$$

Clearly, from the asymptotic growth of $F_{m}$ and $\psi$, the limit in (3.6) goes into the integral. Also, noting that

$$
\begin{gathered}
\lim _{m \rightarrow \infty} F_{m}(\tau)=\lim _{m \rightarrow \infty}\left\langle f_{m}(x), P_{-\frac{1}{2}+i \tau}(\cosh x)\right\rangle \\
=\left\langle f(x), P_{-\frac{1}{2}+i \tau}(\cosh x)\right\rangle=F(\tau),
\end{gathered}
$$

the result holds.

\section{A Paley-Wiener type theorem}

The following theorem proves that any smooth function which satisfies conditions of type (3.4) and (3.5) is the Mehler-Fock transformation of an element of $V^{\prime}$.

Theorem 4.1. Let $F(\tau)$ be a smooth function on I such that there exist a nonnegative integer $r$ and a constant $C$ with $|F(\tau)| \leq C \cdot\left(1+\tau^{2 r}\right)$ for all $\tau>0$. Then there exists a $f \in V^{\prime}$ such that

$$
\left\langle\mathcal{L}^{\prime} f, \psi\right\rangle=\int_{0}^{+\infty} F(\tau) \psi(\tau) d \tau, \forall \psi \in W .
$$

Moreover, for all $\phi \in V$,

$$
\langle f, \phi\rangle=\lim _{T \rightarrow \infty}\left\langle\int_{0}^{T} \tau \tanh (\pi \tau)(\sinh x) P_{-\frac{1}{2}+i \tau}(\cosh \tau) F(\tau) d \tau, \phi(x)\right\rangle .
$$

Proof. Let $G(\tau)$ be a smooth function on $(0, \infty)$ such that

$$
|G(\tau)| \leq M_{1} \cdot\left(1+\tau^{-\alpha}\right),
$$

where $\alpha>2$ and $M_{1}$ is a real constant. Let us define, for any $x>0$,

$$
g(x)=\int_{0}^{\infty} \tau \tanh (\pi \tau)(\sinh x) P_{-\frac{1}{2}+i \tau}(\cosh x) G(\tau) d \tau .
$$

Note that for $\tau \rightarrow 0$,

$$
\tau \tanh (\pi \tau)=O\left(\tau^{2}\right)
$$

and for $\tau \rightarrow \infty$,

$$
\tau \tanh (\pi \tau)=O(\tau)
$$

On the other hand,

$$
\left|P_{-\frac{1}{2}+i \tau}(\cosh x)\right| \leq\left|P_{-\frac{1}{2}}(\cosh x)\right|, \quad \forall x>0, \quad \forall \tau>0 .
$$


So, (4.2) exists and it is a locally integrable function on $I$. Moreover,

$$
\begin{gathered}
\int_{0}^{\infty}\left|(\sinh x)^{-1} g(x)\right| d x=\int_{0}^{\infty}\left|\int_{0}^{\infty} \tau \tanh (\pi \tau) P_{-\frac{1}{2}+i \tau}(\cosh x) G(\tau) d \tau\right| d x \\
\leq \int_{0}^{\infty}\left|P_{-\frac{1}{2}}(\cosh x)\right| \int_{0}^{\infty} \tau|\tanh (\pi \tau)||G(\tau)| d \tau d x \\
\leq M_{2} \cdot \int_{0}^{\infty}\left|P_{-\frac{1}{2}}(\cosh x)\right| d x<\infty
\end{gathered}
$$

where $M_{2}$ is a real constant.

Therefore, the function $g$ yields to a regular member in $V^{\prime}$, denoted by $T_{g}$, which is given by

$$
\left\langle T_{g}, \phi\right\rangle=\int_{0}^{\infty} g(x) \phi(x) d x, \quad \forall \phi \in V .
$$

Also for any $\psi \in W$ denoting $\phi=\mathcal{L} \psi$, and using Lemma 3.2, one obtains

$$
\begin{gathered}
\left\langle\mathcal{L}^{\prime} T_{g}, \psi\right\rangle=\left\langle T_{g}, \mathcal{L} \psi\right\rangle=\int_{0}^{\infty} g(x) \phi(x) d x \\
=\int_{0}^{\infty} \phi(x) \int_{0}^{\infty} \tau \tanh (\pi \tau)(\sinh x) P_{-\frac{1}{2}+i \tau}(\cosh x) G(\tau) d \tau d x
\end{gathered}
$$

which, by Fubini's theorem, is equal to

$$
\int_{0}^{\infty} G(\tau) \int_{0}^{\infty} \tau \tanh (\pi \tau)(\sinh x) P_{-\frac{1}{2}+i \tau}(\cosh x) \phi(x) d x d \tau=\int_{0}^{\infty} G(\tau) \psi(\tau) d \tau .
$$

Thus, for all $\psi \in W$,

$$
\left\langle\mathcal{L}^{\prime} T_{g}, \psi\right\rangle=\int_{0}^{\infty} G(\tau) \psi(\tau) d \tau
$$

Now, for all $\tau>0$, set

$$
G(\tau)=\frac{F(\tau)}{\left(\frac{1}{4}+\tau^{2}\right)^{r+p}} .
$$

Clearly, for $p>\alpha / 2$, this function $G$ satisfies condition (4.1). So, there exists a function $g$ given by (4.2) such that the regular member $T_{g}$ in $V^{\prime}$ satisfies (4.3). Set $f=\left(-A_{x}^{\prime}\right)^{r+p} T_{g}$. Note that, for all $\phi \in V$,

$$
\langle f, \phi\rangle=\left\langle\left(-A_{x}^{\prime}\right)^{r+p} T_{g}, \phi\right\rangle=\int_{0}^{\infty} g(x)\left(-A_{x}\right)^{r+p} \phi(x) d x .
$$

Thus, for all $\psi \in W$, one obtains

$$
\begin{gathered}
\left\langle\mathcal{L}^{\prime} f, \psi\right\rangle=\left\langle T_{g},\left(-A_{x}\right)^{r+p}(\mathcal{L} \psi)\right\rangle=\left\langle T_{g}, \mathcal{L}\left[\left(\frac{1}{4}+\tau^{2}\right)^{r+p} \psi(\tau)\right]\right\rangle \\
=\int_{0}^{\infty} G(\tau)\left(\frac{1}{4}+\tau^{2}\right)^{r+p} \psi(\tau) d \tau=\int_{0}^{\infty} F(\tau) \psi(\tau) d \tau .
\end{gathered}
$$

On the other hand, for any $\phi \in V$,

$$
\langle f, \phi\rangle=\left\langle\int_{0}^{\infty} \tau \tanh (\pi \tau)(\sinh x) P_{-\frac{1}{2}+i \tau}(\cosh x) G(\tau) d \tau,\left(-A_{x}\right)^{r+p} \phi(x)\right\rangle
$$




$$
\begin{gathered}
=\lim _{T \rightarrow \infty}\left\langle\int_{0}^{T} \tau \tanh (\pi \tau)(\sinh x) P_{-\frac{1}{2}+i \tau}(\cosh x) G(\tau) d \tau,\left(-A_{x}\right)^{r+p} \phi(x)\right\rangle \\
=\lim _{T \rightarrow \infty}\left\langle\int_{0}^{T} \tau \tanh (\pi \tau)\left(-A_{x}^{\prime}\right)^{r+p}\left[(\sinh x) P_{-\frac{1}{2}+i \tau}(\cosh x)\right] G(\tau) d \tau, \phi(x)\right\rangle \\
=\lim _{T \rightarrow \infty}\left\langle\int_{0}^{T} \tau \tanh (\pi \tau)(\sinh x) P_{-\frac{1}{2}+i \tau}(\cosh x)\left(\frac{1}{4}+\tau^{2}\right)^{r+p} G(\tau) d \tau, \phi(x)\right\rangle \\
=\lim _{T \rightarrow \infty}\left\langle\int_{0}^{T} \tau \tanh (\pi \tau)(\sinh x) P_{-\frac{1}{2}+i \tau}(\cosh x) F(\tau) d \tau, \phi(x)\right\rangle .
\end{gathered}
$$

\section{MEHLER-Fock TRANSFORMS OF ORDER $n$}

Starting from the integral representation $[1,(11)$, p. 156], which is valid for all $n \in \mathbb{N} \cup\{0\}$, all $\tau>0$ and all $x>0$,

$$
\begin{aligned}
& P_{-\frac{1}{2}+i \tau}^{n}(\cosh x) \\
& \quad=\sqrt{\frac{2}{\pi}} \frac{\Gamma\left(n+\frac{1}{2}\right)(\sinh x)^{n}}{\Gamma\left(n+\frac{1}{2}+i \tau\right) \Gamma\left(n+\frac{1}{2}-i \tau\right)} \int_{0}^{\infty}(\cosh x+\cosh \xi)^{-n-\frac{1}{2}} \cos (\tau \xi) d \xi,
\end{aligned}
$$

the results of the previous sections are readily extended to the so-called MehlerFock transform of order $n \in \mathbb{N}$, the kernel of which is given by $P_{-\frac{1}{2}+i \tau}^{n}(\cosh x)$, the associated Legendre function of first kind and order $n$.

Specifically, one introduces the corresponding spaces $V$ and $W$; namely, $V$ is the vector space consisting of all complex-valued functions $\phi$ which possess derivatives of all orders on $I$ and such that, for all $k \in \mathbb{N} \cup\{0\}$,

$$
\gamma_{k}(\phi)=\sup _{x \in I}\left|(\sinh x) A_{x}^{k} \phi(x)\right|<\infty,
$$

where $A_{x}$ is the differential operator

$$
A_{x} \equiv(\sinh x)^{-n-1} D_{x}(\sinh x)^{2 n+1} D_{x}(\sinh x)^{-n},
$$

and $W$ is the vector space of all complex-valued even functions $\psi$ on $\mathbb{R}$ such that

$$
\frac{\left(\left(n+\frac{1}{2}\right)^{2}+\tau^{2}\right)^{r} \psi(\tau)}{\tau \Gamma\left(n+\frac{1}{2}+i \tau\right) \Gamma\left(n+\frac{1}{2}-i \tau\right)} \in L^{1}(\mathbb{R})
$$

and

$$
\rho_{r}(\psi)=\sup _{x \in I}\left|x^{\frac{3}{2}} e^{x}\left(\mathcal{F}\left[\frac{\left(\left(n+\frac{1}{2}\right)^{2}+\tau^{2}\right)^{r} \psi(\tau)}{\tau \Gamma\left(n+\frac{1}{2}+i \tau\right) \Gamma\left(n+\frac{1}{2}-i \tau\right)}\right]\right)(x)\right|<\infty,
$$

for all $r \in \mathbb{N} \cup\{0\}$, and $\mathcal{F}$ denotes Fourier transform with respect to the $\tau$-variable.

The operator $\mathcal{L}: W \rightarrow V$, given by

$$
(\mathcal{L} \psi)(x)=\int_{0}^{\infty} P_{-\frac{1}{2}+i \tau}^{n}(\cosh x) \psi(\tau) d \tau
$$

becomes a continuous linear mapping from $W$ in $V$. 
Also, for any $\phi \in V$ and all $\tau>0$ set

$$
\begin{aligned}
\psi_{\phi}(\tau)= & \frac{1}{\pi} \tau \sinh (\pi \tau) \Gamma\left(n+\frac{1}{2}+i \tau\right) \Gamma\left(n+\frac{1}{2}-i \tau\right) \\
& \times \int_{0}^{\infty}(\sinh x) P_{-\frac{1}{2}+i \tau}^{n}(\cosh x) \phi(x) d x .
\end{aligned}
$$

It is obtained that

i) $\psi_{\phi}(\tau)=O\left(\tau^{2 n+2}\right)$, as $\tau \rightarrow 0$;

ii) for all $p \in \mathbb{N}, \psi_{\phi}(\tau)=O\left(\tau^{-p}\right)$, as $\tau \rightarrow \infty$.

Also, for any $\psi \in W$ there exists $\phi \in V$ such that $\psi=\psi_{\phi}$.

Now, for any $f \in \mathcal{E}^{\prime}(I)$, the function

$$
F(\tau)=\left\langle f(x), P_{-\frac{1}{2}+i \tau}^{n}(\cosh x)\right\rangle, \quad \tau>0,
$$

satisfies

i) $F(\tau)=O(1)$, as $\tau \rightarrow 0$;

ii) there exists $r \in \mathbb{N} \cup\{0\}$ such that $F(\tau)=O\left(\tau^{2 r}\right)$, as $\tau \rightarrow \infty$.

Thus the corresponding result of Theorem 3.1 holds in this setting; namely, for all $f \in \mathcal{E}^{\prime}(I)$,

$$
\left\langle\mathcal{L}^{\prime} f, \psi\right\rangle=\int_{0}^{\infty} F(\tau) \psi(\tau) d \tau
$$

Also the corresponding Paley-Wiener type theorem establishes that

Theorem 5.1. Let $F(\tau)$ be a smooth function on I such that there exist a nonnegative integer $r$ and a constant $C$ with $|F(\tau)| \leq C \cdot\left(1+\tau^{2 r}\right)$ for all $\tau>0$. Then there exists an $f \in V^{\prime}$ such that

$$
\left\langle\mathcal{L}^{\prime} f, \psi\right\rangle=\int_{0}^{+\infty} F(\tau) \psi(\tau) d \tau, \quad \forall \psi \in W .
$$

Moreover, for all $\phi \in V$,

$$
\langle f, \phi\rangle=\lim _{T \rightarrow \infty}\left\langle\int_{0}^{T} S(\tau) G(\tau, x) F(\tau) d \tau, \phi(x)\right\rangle,
$$

where

$$
S(\tau)=\frac{1}{\pi} \tau \sinh (\pi \tau) \Gamma\left(n+\frac{1}{2}+i \tau\right) \Gamma\left(n+\frac{1}{2}-i \tau\right)
$$

and

$$
G(\tau, x)=(\sinh x) \cdot P_{-\frac{1}{2}+i \tau}^{-n}(\cosh x)
$$

\section{REFERENCES}

1. A. Erdélyi, W. Magnus, F. Oberhettinger and F. Tricomi, Higher transcendental functions, Vol. I, McGraw-Hill, New York, 1953. MR 15:419i

2. V. Fock, On the representation of an arbitrary function by an integral involving Legendre's function with a complex index, Dokl. Akad. Nauk. SSSR 39 (1943), 253-256. MR 5:181b

3. H.N. Glaeske and A. Hess, On the convolution theorem of the Mehler-Fock transform for a class of generalized functions (I), Math. Nachr. 131 (1987), 107-117. MR 88k:46045

4. H.N. Glaeske and A. Hess, On the convolution theorem of the Mehler-Fock transform for a class of generalized functions (II), Math. Nachr. 136 (1988), 119-129. MR 89j:46042

5. N. Hayek and B.J. González, On the Mehler-Fock transform of generalized functions, Bull. Soc. Roy. Sci. Liège 61 (3-4) (1992), 315-327. MR 93g:44013 
6. J. Horváth, Topological Vector Spaces and Distributions, Vol. I, Addison-Wesley, Reading, MA, 1966. MR 34:4863

7. B. Lisena, On the Generalized Kontorovich-Lebedev Transform, Rend. Mat. Appl. 9 (VII) (1989), 87-101. MR 91c:44005

8. R.S. Pathak and R.K. Pandey, The generalized Mehler-Fock transformation of distributions, Arabian J. Sci. Engrg. 10 (1) (1985), 39-57. MR 86b:44005

9. D.B. Sumner, An inversion formula for the generalized Stieltjes transform, Bull. Amer. Math. Soc. 55 (1949), 174-183. MR 10:370f

10. U.N. Tiwari and J.N. Pandey, The Mehler-Fock transform of distributions, Rocky Mountain J. Math. 10 (1980), 401-408. MR 81m:46058

11. F. Treves, Topological Vector Spaces, Distributions and Kernels, Academic Press, New York, 1967. MR 37:726

Departamento de Análisis Matemático, Universidad de La Laguna, 38271 Canary IsLANDS, SPAIN

E-mail address: bjglez@ull.es

E-mail address: enegrin@ull.es 